\title{
An energy-efficient low-memory image compression system for multimedia loT products
}

\author{
Seong-Won Lee* and Ho-Young Kim
}

\begin{abstract}
Emerging Internet of things (IoT) technologies have rapidly expanded to multimedia applications, including highresolution image transmission. However, handling image data in loT products with limited battery capacity requires low-complexity and small-size solutions such as low-memory compression techniques. The objective of this paper is to propose a line-based compression system based on four-level two-line discrete wavelet transform and adaptive line prediction. Bit stream is generated by multiplexing various frequency components with run-level coding combined with Huffman coding. The proposed system also includes a new bit rate control algorithm that could significantly improve image quality consistency in one frame. The proposed low-memory compression system can retain image quality for visually lossless compression criteria over the whole image frame. It can simultaneously lower total system power consumption in multimedia loT products better than other existing low-memory compression techniques.
\end{abstract}

Keywords: Multimedia Internet of things, Line-based low-memory compression, Image quality consistent bit rate control

\section{Introduction}

With exciting development of very large-scale integration (VLSI) technology, Internet of things (IoT) products have rapidly evolved into monitoring devices that can capture and stream multimedia data with high-resolution images. Promising multimedia IoT applications include smart glasses [1, 2], unmanned aerial vehicles [3], telepresence robots [4], and wireless capsule endoscopies [5] that can capture and transmit still images or video sequences at visually lossless levels. However, processing a high volume of image data and wirelessly transmitting data in a multimedia IoT device that typically has limited battery capacity require high-power consumption. This is a major obstacle to system operation time.

In multimedia IoT devices, key factors of system power consumption include a microprocessor for information processing, memory devices for data storage, and wireless transmitter-receivers for data transmission. The amount of power consumed by the memory device is an important factor when transmitting and processing a

\footnotetext{
* Correspondence: swlee@kw.ac.kr

Department of Computer Engineering, Kwangwoon University, 20, Gwangun-ro, Nowon-gu, Seoul, Republic of Korea
}

high volume of data such as images. Therefore, many studies have been conducted to reduce power consumption by decreasing the size of image data with simple compression method before transmission to use computing processes and memory as small as possible [6-8]. Accordingly, low-memory compression methods have been adopted in mobile devices, especially in those that require high-power consumption and bandwidth for high-resolution display [9] and in vehicle safety systems that require very low delays for real-time image transmission [10].

In addition to image data, low-memory compression methods have been applied to body signal monitoring IoT devices such as two-dimensional (2D) electrocardiogram (ECG) recorders using high temporal correlations between ECG signals by converting 1D ECG signals into 2D ECG signals, thus reducing amounts of data transmitted [11].

Existing image compression standards such as JPEG2K [12], H.264 [13, 14], and HEVC [15, 16] can reduce the power required for transmission while maintaining a high compression ratio. However, power consumption of these standards is increased in the processor and 
memory due to vast amounts of processing operations and extremely large storage devices. Thus, less complex JPEG standard [17] is often applied to conventional multimedia Io $\mathrm{T}$ products to compress a whole single frame. However, the JPEG standard does not easily satisfy the demand for high image quality due to its fixed-point implementation. To achieve visually lossless condition which means more than $40-\mathrm{dB}$ reconstruction image quality, the JPEG standard requires a floating-point processing unit that consumes a lot of power. In addition, for a high-resolution image with a visually lossless image where information of minute size is important, a line error is advantageous for recovering information rather than an error of a block occurring in conventional block-based compression standard methods. Another requirement for multimedia IoT devices is that a compact compression technique with small physical size and small production cost is often more important than high compatibility, because the IoT devices are often used in dedicated applications which often require very high volume of small-size devices.

One-dimensional (1D) set partitioning in hierarchical trees (SPIHT) technique based on zerotree wavelet coding has been presented in a low-memory compression study [18]. However, 1D SPIHT memory access counts are too high during the process of repetitively arranging key data in bit manipulations after wavelet transform for transmission, resulting in excessive power consumption. Several studies have been conducted to reduce memory accesses and improve compression ratio of 1D SPIHT [19-22], including line-based backward coding of wavelet trees (L-BCWT) $[23,24]$ and zero memory set partitioned embedded block (ZM-SPECK) [25]. However, energy efficiency of systems after adopting these modified SPIHT methods needs to be further improved to satisfy energy requirements of low-power IoT devices.

Vo et al. [26] have conducted a study on low-memory compression method after applying non-uniform quantization techniques according to block types by considering error perception difference characteristics of human visual system (HVS). They achieved a compression ratio of 3:1. Kim et al. [27] have also presented a compression system that can perform horizontal predictive coding for low-frequency band with zero-zone scalar quantization techniques for higher frequencies after a line-based four-level discrete wavelet transform (DWT). After applying variable length coding method, they achieved a compression ratio of approximately 3:1 with a visually lossless condition.

The objective of this study was to propose a low-memory compression method to reduce overall power consumption of multimedia IoT products. The proposed compression method includes a compression method for compressing a single frame image and a bit rate control method for maintaining compression ratio and consistency of image quality through the image. This method extends and improves the existing frequency adaptive line compression (FALC) technique of Kim et al. [27], achieving a higher compression ratio by using the least possible amount of processing and memory. The proposed technique adopts 2D DWT and 2D directional adaptive differential pulse code modulation (DPCM) between lines. A combination of run-level coding and Huffman coding further improves the compression ratio of high-frequency data. The bit rate control method coupled with the proposed system maintains the consistency of image quality through the frame mostly with the information collected in the current frame. However, the method also checks if the current frame is similar to the previous frame as a part of an image sequence, because additional improvement of image quality can be easily achieved by using a simple information like a type of image. It can significantly alleviate the issue of image quality imbalance that might occur between highs and lows in a single image, an inevitable defect in low-memory compression methods. The proposed technique can achieve a compression ratio of at least 4:1 with an average peak signal-to-noise ratio (PSNR) of $40 \mathrm{~dB}$.

In Section 2 of this paper, background information and conventional compression methods are introduced. The proposed compression system is described in Section 3. In Section 4, the method is evaluated and results are discussed. Section 5 concludes this paper.

\section{Background research}

\subsection{Energy efficiency of compression methods for loT system}

With rapid development of IoT technology recently, securing sufficient operation time for IoT products with limited battery capacities has become an important performance index. Basic components of an applied IoT system include batteries as the main power source, sensors to acquire data, memory to store acquired data, microprocessors to process stored data, and transmission devices to transmit processed data wirelessly as shown in Fig. 1.

Applications of recent IoT devices are being expanded to multimedia. Such devices can capture and stream large volumes of image data. However, storing, processing, and wirelessly transmitting huge amounts of image data will increase power consumption and further reduce operation time of the overall system.

Image compression standard techniques such as JPEG2K, H.264, and HEVC have been used to reduce the size of image data. Although transmission power can be significantly reduced by using high compression ratios, high complexity of the compression system also 


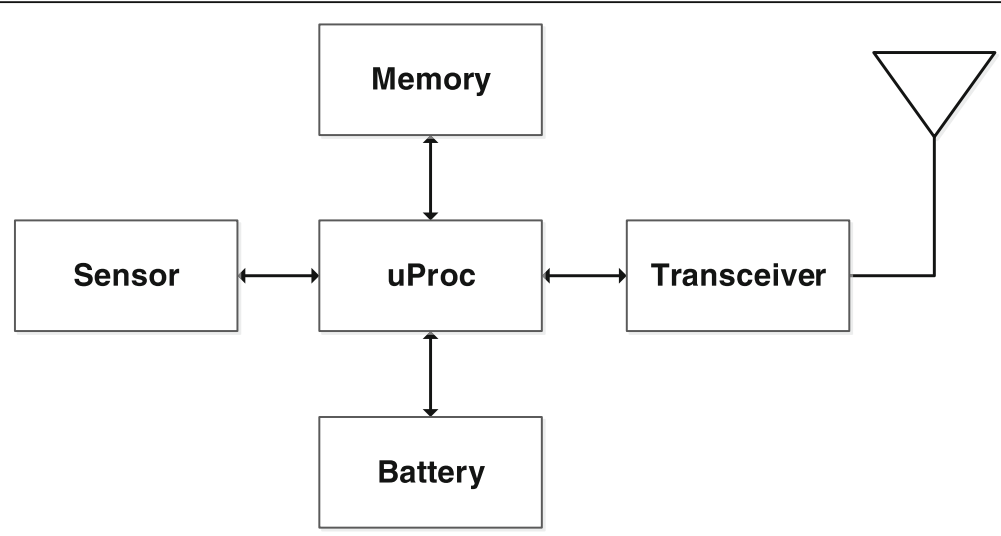

Fig. 1 Basic compositions of loT applied systems (uProc, microprocessor)

increases power consumption due to excessive processing and memory demands in the compression process. Hence, many studies have been conducted on energy-effective compression methods based on low memory to reduce power consumption of multimedia IoT systems [6-8]. These methods can further reduce power in the overall system and extend the operation time of batteries by lowering processing operations and memory accesses for the compression system.

Figure 2 shows comparison of estimated power consumption of several compression methods on a multimedia IoT platform (Intel Galileo gen 2 development board) [28]. Cases were assumed to involve the application of image data monitoring. 4L H.264 is an intra compression mode based on H.264 main profile [13] with joint model (JM) 19.0 reference software [14] where coding unit size is fixed to 4 by 4 to use only four lines and all functions that include nine intra prediction modes and context-adaptive arithmetic coding (CABAC). Likewise, $4 \mathrm{~L}$ HEVC is an intra compression mode based on HEVC main profile [15] with HEVC test model (HM) 16.5 reference software [16] where coding unit size is also fixed to 4 by 4 to use only four lines and all functions that include 35 intra prediction modes and CABAC. As shown in Fig. 2, power consumption of the whole IoT system is increased for 4L H.264 and 4L HEVC than 1D SPIHT.

\subsection{Conventional compression algorithms}

The 1D SPIHT method [18] is based on zerotree characteristics of wavelet coefficients. To enhance compression efficiency, this method generates three dynamic lists by repeatedly traversing coefficients from the upper bit plane to the lower bit plane to transmit important bit data first. This method is robust to transmission errors and easy to control bit rate. However, the 1D SPIHT compression method incurs high-power consumption due to its increased memory accesses during the repetitive process of managing three dynamic lists based on bit-plane coding.

L-BCWT method [23] can lower the complexity of existing 1D SPIHT by using a lookup table method called a one-pass backward coding technique. Although L-BCWT can reduce the complexity of repeated tree-scanning, bit-plane coding, and dynamic lists management of conventional 1D SPIHT, its compression performance remains marginal.

ZM-SPECK [25] can remove state-maps and dynamic lists in the existing SPIHT algorithm using linear indexing property of wavelet tree and merged refinement technique. This method can reduce both computational complexity and memory accesses related to dynamic lists. However, it still has a lot of memory accesses due to its bit-plane coding and recursive set-partition technique. The compression ratio of ZM-SPECK is slightly higher than that of the existing SPIHT algorithm.

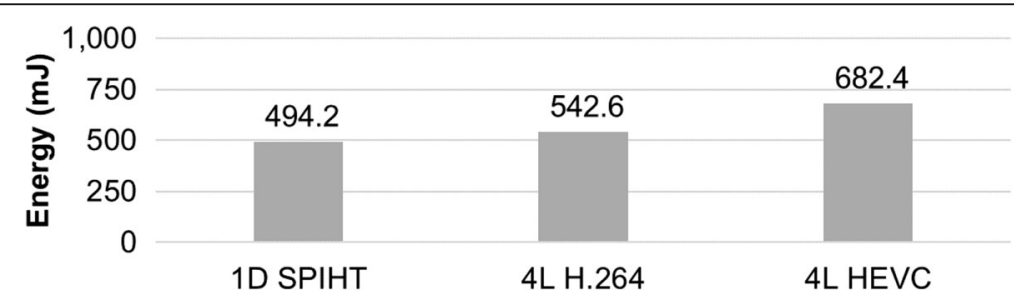

Fig. 2 Energy comparison of 1D SPIHT, 4L H.264, and 4L HEVC methods 


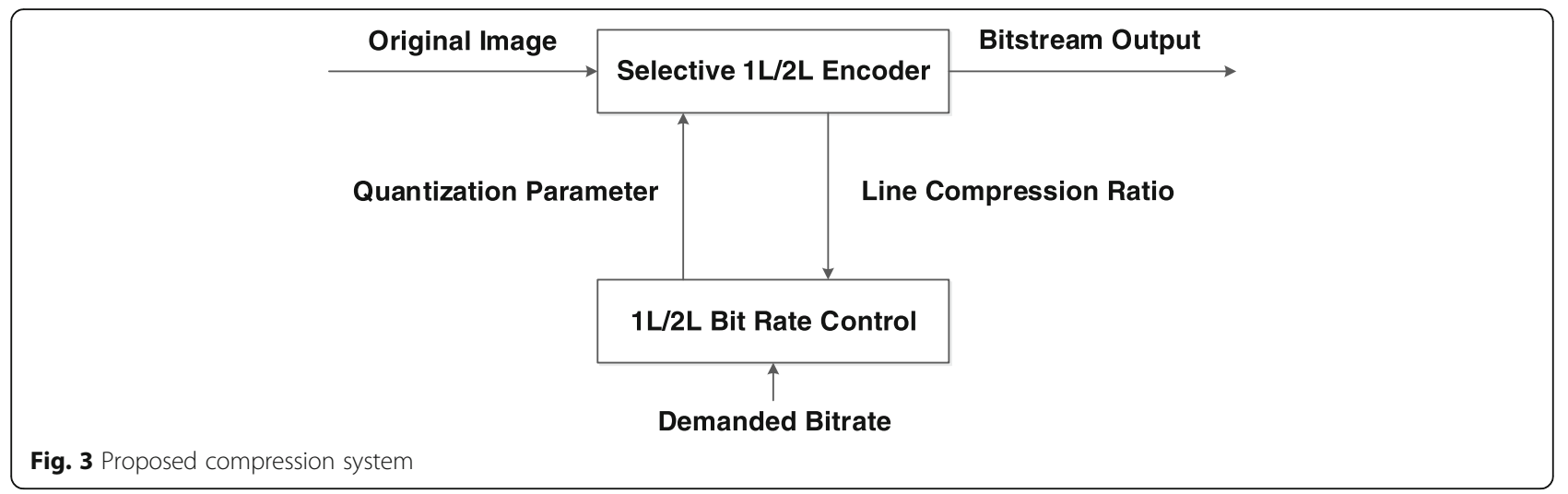

Vo et al. [26] have proposed a line compression method called visually lossless compression non-uniform quantization (VLC_NUQ) method. This method can perform median edge detection (MED) prediction of $2 \times$ 4 blocks in areas where errors are relatively less recognizable. It can also perform MED prediction of $1 \times$ 4 blocks in detailed and flat areas where errors are easily noticeable. Values of each block are transmitted to the decoder after data are further reduced through predefined non-uniform quantization process. Although the simplicity of VLC_NUQ method only requires small amounts of power consumption, its average compression ratio of 3:1 is insufficient for reducing transmission power in applied IoT systems.

Kim et al. [27] have proposed a frequency adaptive line compression (FALC) method with decreased complexity comparing to existing compression methods. Instead of using bit planes of RGB data, FALC method uses whole coefficients of discrete wavelet transforms of $\mathrm{YCuCv}$ color space data. After four levels of wavelet transforms, the original data are transformed into four high-frequency bands and one low-frequency band. Then, selective zero-zone quantization is performed in the four high-frequency bands. For the low-frequency band, predictive coding removes redundancy. Data from each frequency band are then compressed with a variable length coding (VLC) method based on Huffman coding. The compression ratio of FALC surpasses 3:1 with a visually lossless condition. However, further improvement is needed to actively reduce more power in IoT-applied environments.

\subsection{Existing line-based bit rate controls}

The image quality of line-based compression methods often fluctuates over a single frame because each line even in a single frame has different characteristics in terms of compression efficiency. In JPEG-LS environment, Edirisinghe's line-based bit rate control (BRC) [29] and Jiang's line-based BRC [30] have been proposed. However, these BRCs have insufficient image qualities in terms of consistency within one frame.

Another bit rate control method [31] has been proposed to improve the consistency of image quality within one frame based on the existing FALC method. It keeps the quantization level change slow. It also checks

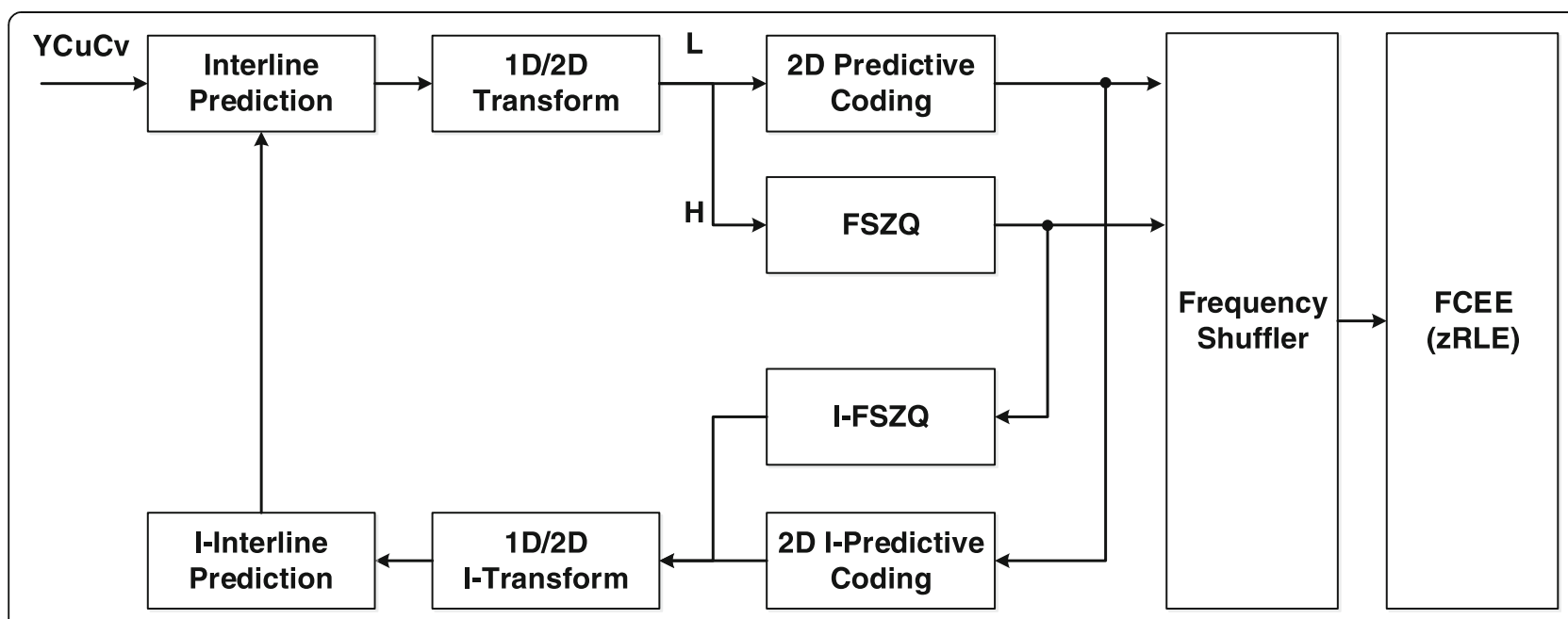

Fig. 4 Proposed $1 \mathrm{~L} / 2 \mathrm{~L}$ selective encoder system 


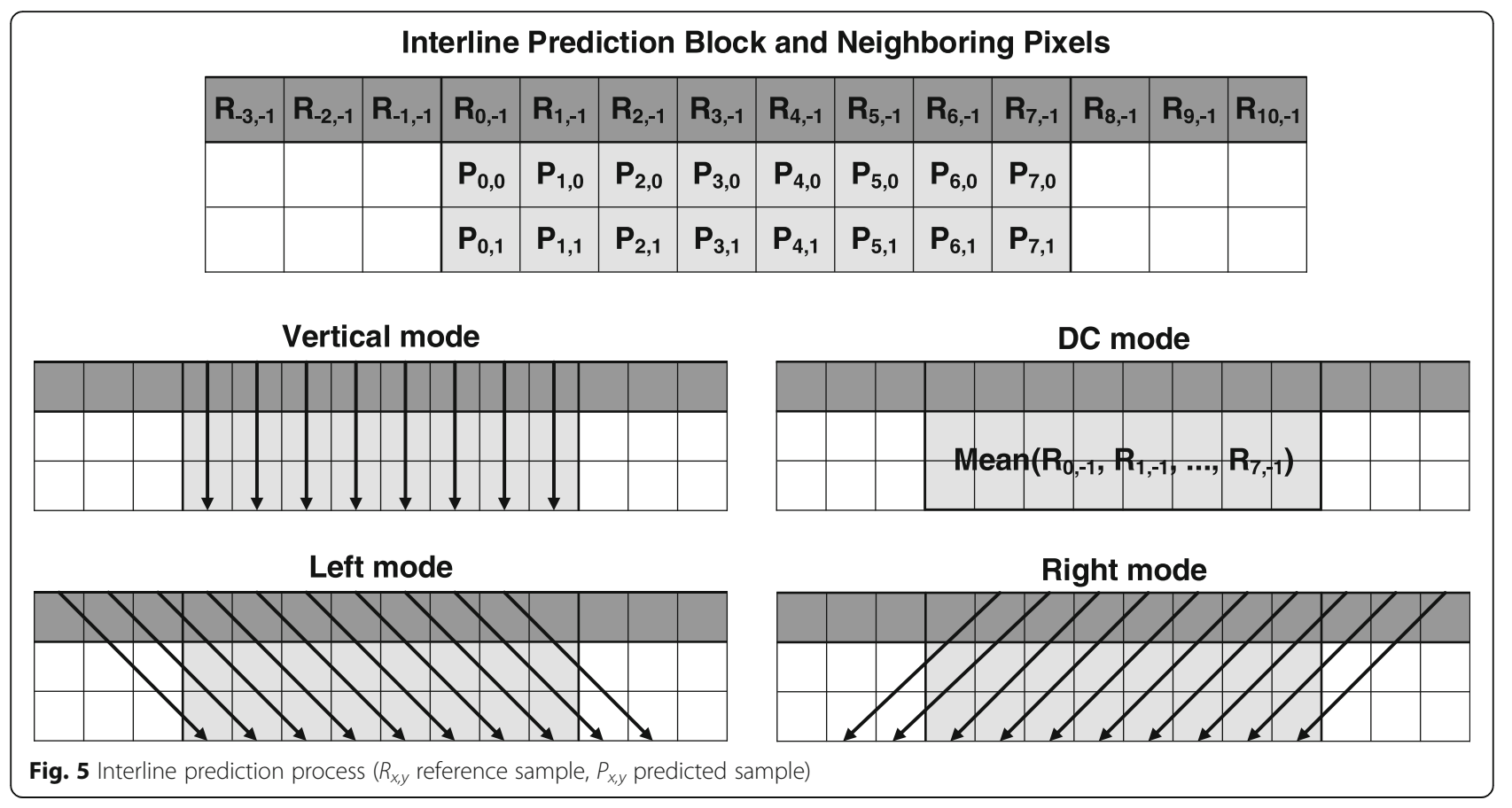

image quality and available bits for the rest of the frame when two thirds of the frame is processed to find an appropriate quantization level that will use up the remaining bits.

However, existing BRC methods still have insufficient image quality consistency, especially when there are image splits or scene changes. Therefore, BRC must be further improved to be applied to consumer products.

\section{Proposed compression system}

The proposed compression system includes a compression method based on two consecutive lines and a bit rate control system to ensure the compression ratio for a whole frame. Figure 3 shows the diagram of the proposed compression system. To achieve higher power efficiency of the compression method, we extended the existing FALC method to improve the efficiency of each processing stage. To improve consistent image quality through the entire frame, bit rate control system is used to check characteristic variations of the frame.

\subsection{The proposed selective $1 \mathrm{~L} / 2 \mathrm{~L}$ compression method}

The proposed selective $1 \mathrm{~L} / 2 \mathrm{~L}$ compression method can remove spatial redundancy occurring between adjacent lines in spatial interline prediction after 1D DWT is extended to 2D DWT. To lower power consumption of the proposed compression method, we considered the smallest possible operations and memory accesses. Lastly, run-length encoding for repeated zeros was applied to repetitive zero values in high-frequency bands of chroma channels to further enhance the overall entropy coding compression performance. Overall structure of the proposed encoder is shown in Fig. 4. The following subsections will step by step describe the overall block diagram shown in Fig. 4.

\subsubsection{Spatial interline prediction}

To effectively remove spatial redundancy between lines, a vertical interline predictive method is used. The proposed interline prediction method can calculate several directional sum of absolute difference (SAD) between two adjacent lines and then choose the prediction mode to

Table 1 Proposed interline prediction modes

\begin{tabular}{|c|c|c|c|}
\hline Mode & Prediction eq. (1L mode $1 \times 8$ block, $2 \mathrm{~L}$ mode & $2 \times 8$ block) & Color channel \\
\hline Vertical & $P_{x, y}=P_{x, y+1}=R_{x, y-1}$ & & Luma, chroma \\
\hline Left & $P_{x, y+m}=\left(R_{x-2-m, y-1}+R_{x-1-m, y-1}\right) \gg 1$ & $m=0$ or 1 & Luma \\
\hline DC & $P_{x, y}=P_{x, y+1}=\left(\sum_{i \in B_{x}} R_{i, y-1}\right) \gg 3$ & $B_{x}=\{$ all $x$ of $R\}$ & Luma, chroma \\
\hline Right & $P_{x, y+m}=\left(R_{x+1+m, y-1}+R_{x+2+m, y-1}\right) \gg 1$ & $m=0$ or 1 & Luma \\
\hline
\end{tabular}




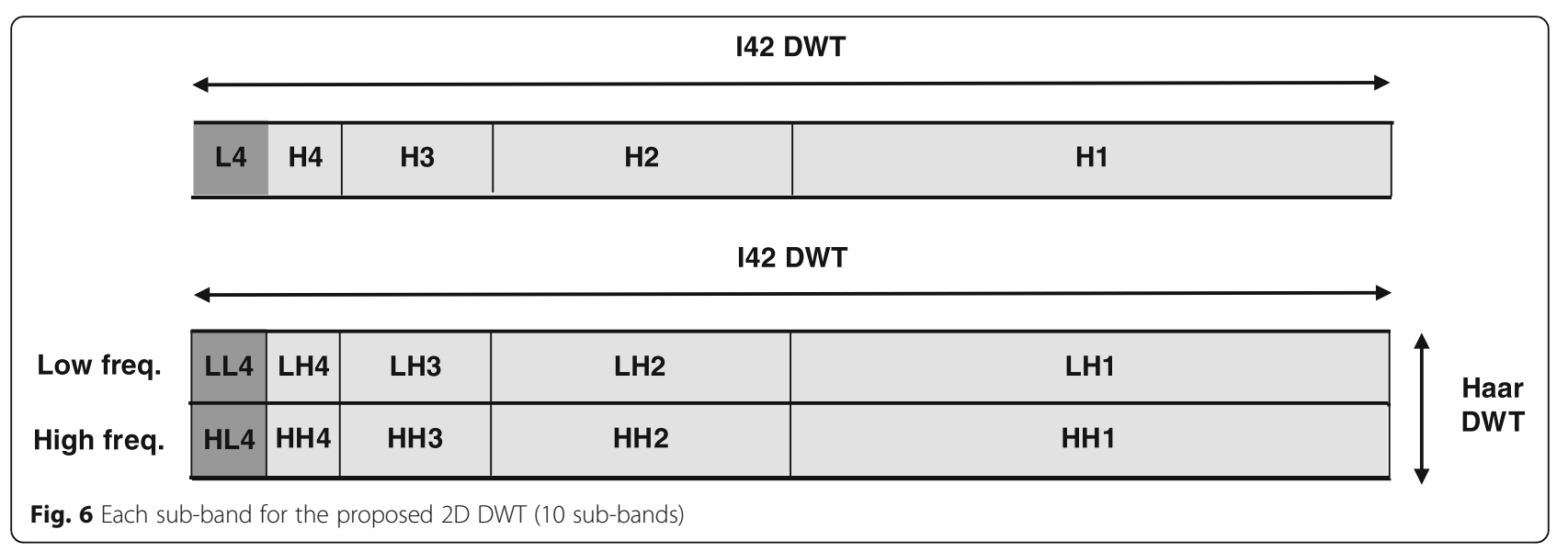

minimize SAD. Four prediction modes are determined by considering both compression performance and transmission cost.

Since luminance channels have relatively higher spatial correlations compared to chroma channels, four spatial modes are defined for luminance channels while there are only two prediction modes for chroma channels. Horizontal prediction for one-line compression mode (1L mode) is processed with a $1 \times 8$ block while vertical two-line compression mode ( $2 \mathrm{~L}$ mode) is done with a $2 \times 8$ block. Figure 5 shows the relationship between reference samples of previous line and predicted samples in processing lines. Predicted candidate modes are shown in Table 1 , where $R_{x, y}$ is the reference sample, $P_{x, y}$ is the predicted sample, and $x, y$ show the location of a pixel.

\subsubsection{D discrete wavelet transform}

For 2D DWT in the 2L mode, a vertical DWT followed by the existing horizontal I42 integer DWT [27] to further remove vertical redundancy between two adjacent lines of an image. To have the minimum system complexity, Haar DWT [32] is used for vertical DWT. After the proposed 2D DWT is applied, there are 10 sub-bands: five for horizontal DWT and two for vertical DWT. Figure 6 shows sub-bands for the proposed 2D DWT.

\subsubsection{Extended frequency selective zero-zone quantization}

In the existing low power line compression method, Ham et al. [31] have expanded 16 quantization levels of the existing single line-based line compression study [27] to 89 levels for a more precise bit rate control. In the proposed method, the number of quantization levels is reduced to 80 to decrease transmission cost. An additional 80-level quantization table for 2D DWT coefficients is also included and quantization parameters of each level are defined to be compatible to those of PSNR of the same quantization level in the $1 \mathrm{~L}$ mode quantization table.

\subsubsection{D predictive coding in frequency domain}

To remove remaining data redundancy after interline prediction in the spatial domain, an adaptive predictive coding in the frequency domain is performed. Besides using the existing horizontal predictive coding of FALC, the proposed method attempts to use vertical predictive coding in low-frequency band. The proposed method can predict bit stream sizes based on the VLC table for both horizontal and vertical predictive coding modes. It then selects one that has a small size out of these two predictive coding modes to achieve better compression ratio. We did not apply both prediction modes at the same time because once the horizontal predictive coding

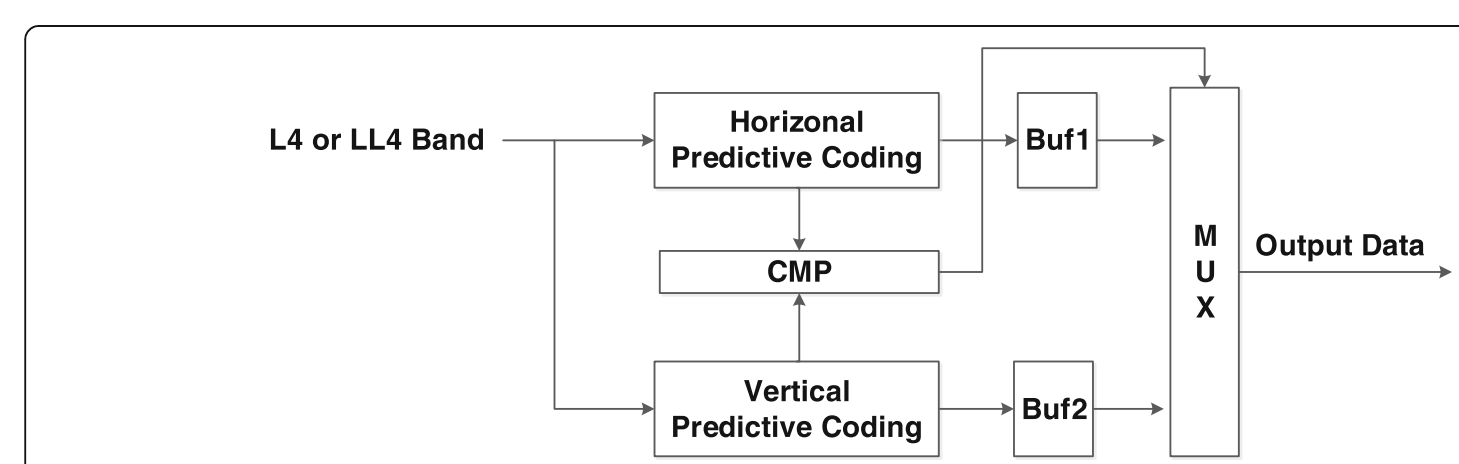

Fig. 7 Optimal predictive coding selection process (horizontal coding or vertical coding) 


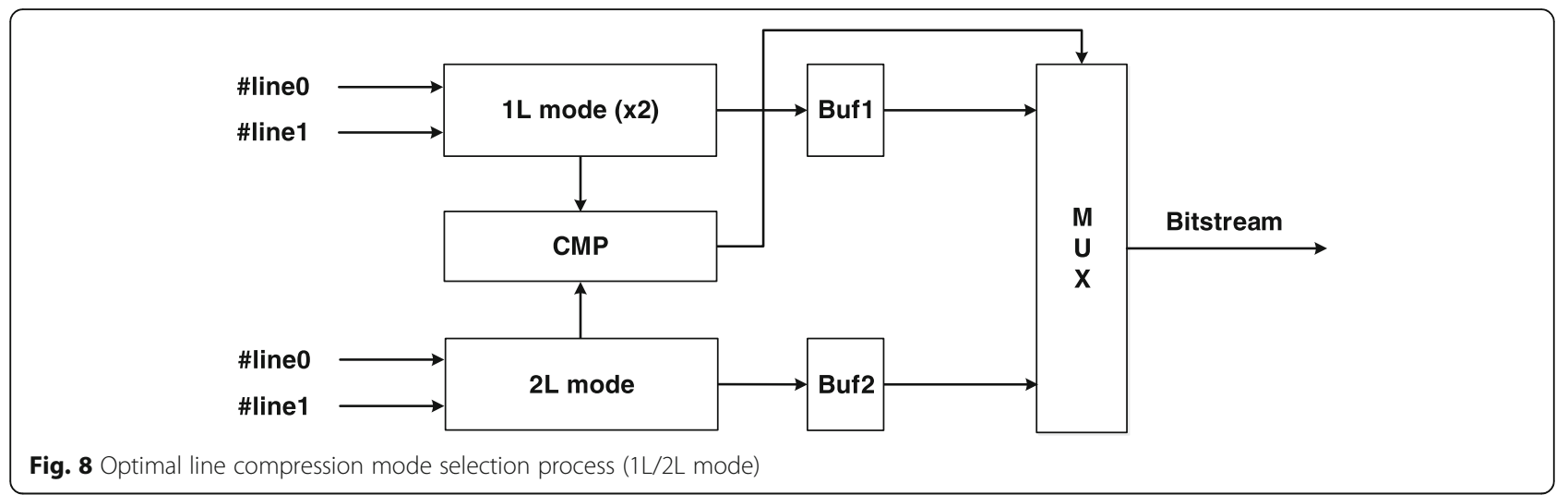

was applied, differential values had less correlations in the vertical direction. The optimal predictive coding selection process is shown in Fig. 7.

\subsubsection{Frequency component entropy encoding}

In the proposed $1 \mathrm{~L} / 2 \mathrm{~L}$ compression method, spatial interline prediction and vertical DWT often produce consecutive zeros in high-frequency chroma channels. The proposed frequency component entropy encoding (FCEE) includes a run-length code for repeated zeros in the chroma channel's H3/2/1 band. The proposed zero run-length encoding (zRLE) composed of a zRLE header and the length of consecutive zeros. The length value is further compressed through independent Huffman coding.

\subsubsection{Output selection process of optimal compression mode}

The proposed method selects two lines of $1 \mathrm{~L}$ mode or one two-line block of $2 \mathrm{~L}$ mode depending on characteristics of input video lines. The $1 \mathrm{~L}$ mode can have better compression performance than the $2 \mathrm{~L}$ mode if the correlation between input lines is low as in complex texture images. On the other hand, the $2 \mathrm{~L}$ mode using the 2D
DWT can have better performance than the $1 \mathrm{~L}$ mode if the correlation between adjacent lines is high.

Figure 8 shows the proposed selection process of optimal compression mode. The proposed method can store two individually compressed image data through $1 \mathrm{~L}$ and 2L compression modes in the temporary output buffer before transmitting data to the receiver. The comparator at the center predicts and compares bits generated by each compression mode to select a more efficient compression method as output value. The proposed method can guarantee a higher compression performance for images with more intense characteristic changes between image lines such as line-based scene changes or text images.

\subsection{Proposed BRC method for $1 \mathrm{~L} / 2 \mathrm{~L}$ compression}

In the existing BRC method for line compression [31], current image type is estimated based on current quantization level (QL) and the resulting line compression ratio (LR). A new QL is then computed for the next line. The relationship between $\mathrm{QL}$ and $\mathrm{LR}$ for each image type is summarized in the BRC table.

Since quantization levels in the $2 \mathrm{~L}$ mode had compatible PSNRs to those in the $1 \mathrm{~L}$ mode, but not compatible LRs, we extend the existing BRC for the proposed BRC

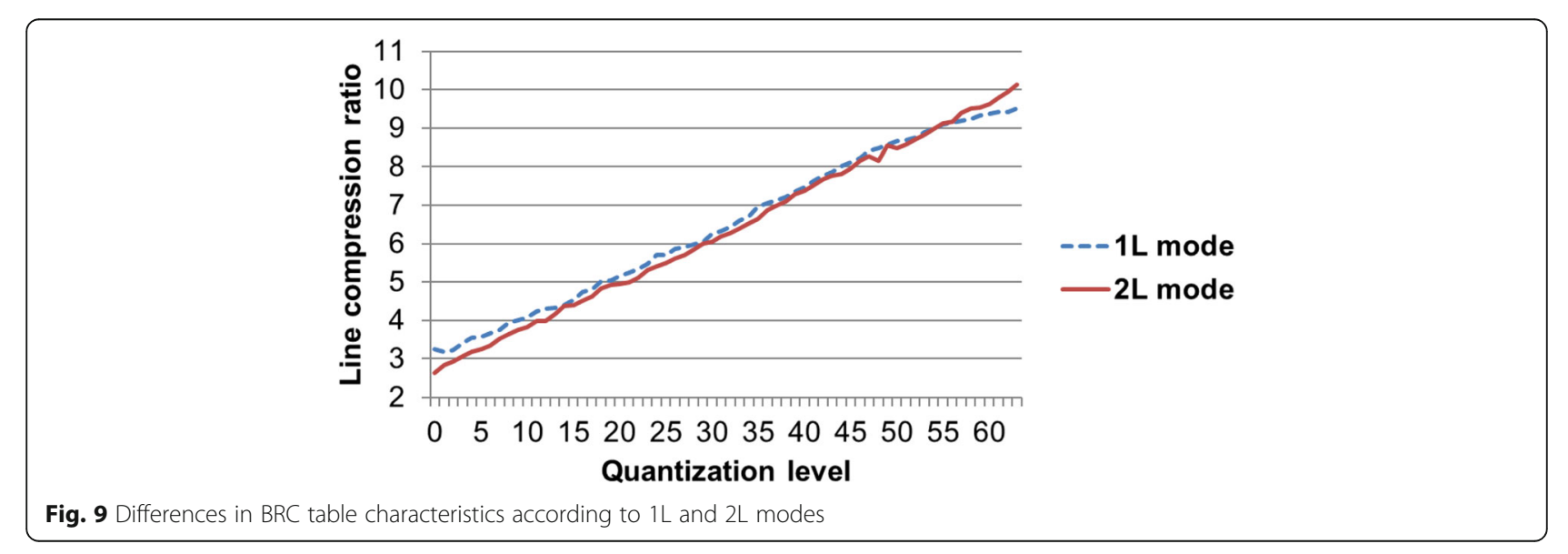


to have both $1 \mathrm{~L}$ BRC table and $2 \mathrm{~L}$ BRC table. The proposed BRC method also includes a compensating method for characteristic differences of linearity between 1L BRC table and 2L BRC table. Figure 9 shows differences of compression performance between $1 \mathrm{~L}$ and $2 \mathrm{~L}$ modes along with quantization levels.

The proposed method also predicts an overall compression outline for the current frame based on average energy of $\mathrm{H} 1$ bands of the previous frame. The previous frame is divided into several subsections, and two middle subsections are analyzed to check if there is a split mode. The proposed BRC method then adjusts target compression ratios (TCRs) for subsections of the current frame. Figure 10 shows an example of a split image mode where general videos and text images are combined.

\subsubsection{BRC algorithm for $1 L / 2 L$ compression}

The proposed BRC determines QL for the next one or two lines from the BRC table based on previously selected compression mode which is either $1 \mathrm{~L}$ or $2 \mathrm{~L}$. The compression mode of the previous line(s) was selected because it had better compression ratio with relatively lower QL of the selected mode. In other words, the QL used for the other mode that was not selected did not achieve enough compression ratio. Therefore, if the BRC decides to increase compression ratio by one step more for the next line and alter the compression mode, the QL of the new mode for the next line(s) should be increased by two steps to meet the demand.

The proposed BRC method has two separate BRC tables for $1 \mathrm{~L}$ and $2 \mathrm{~L}$ modes. It maintains $\mathrm{QL}$ values of both tables for the next lines if either one of two modes is selected. Table 2 shows how quantization level determination (QLD) of the proposed BRC works. As shown in Table 2, the proposed BRC defines two states for the compression ratio variations: (1) stable state for $3.5<\mathrm{LR}<4.5$ and (2) scene change state for others. LR1 and $L R 2$ represent $L R$ for $1 L$ mode and $L R$ for $2 L$ mode, respectively. QL1 and QL2 represent QL values for the next line compression of $1 \mathrm{~L}$ mode and $2 \mathrm{~L}$ mode, respectively. TCR is the target compression ratio for the whole frame.

For example, case 1 in Table 2 shows that BRC is in a stable state while the current compression mode is in $1 \mathrm{~L}$ mode. Since compression result of LR1 is less than TCR in case 1 , next lines should be compressed more. Therefore, quantization levels for next lines QL1 and QL2 are increased by 1 and 2, respectively. When the BRC compresses the next lines, it will compare compression results of $1 \mathrm{~L}$ and $2 \mathrm{~L}$ modes and then chooses one with better result based on the trade-off between compression performance and image quality. For case 2, everything is the same as in case 1 except that LR1 is higher than TCR. In this case, QL1 is decreased by 1 but QL2 will remain the same as before.

In cases with scene change, it is difficult to define QL values for both compression modes. Therefore, a new single QL is selected in accordance with the BRC table for the current compression mode. The new QL is assigned to both $\mathrm{QL}$ values of $1 \mathrm{~L}$ and $2 \mathrm{~L}$ modes because the same QL value for both compression modes has similar image quality in terms of PSNR. Slight quality difference in the QL value can be adjusted through subsequent processing.

If there are a small number of remaining lines to be compressed while the cumulative compression ratio (CR) does not meet TCR, the quantization level for next

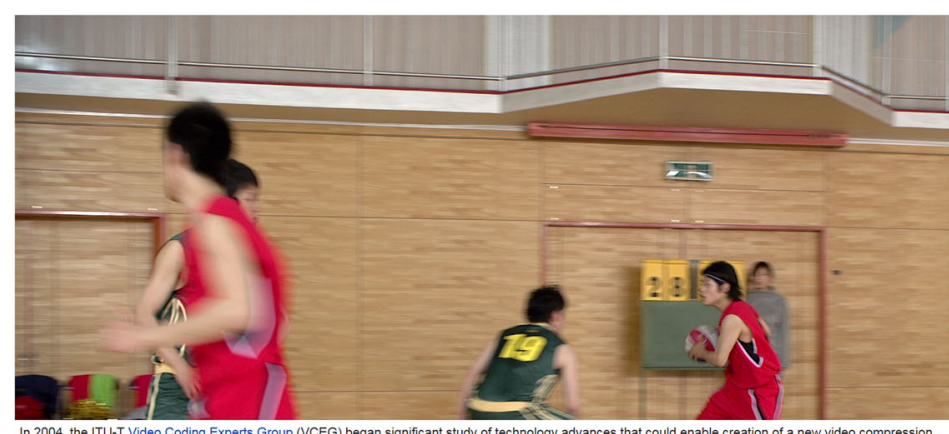

In 2004, the ITU-T Video Coding Experts Group (VCEG) began significant study of technology advances that could enable creation of a new video comprester enhancement of the H.264/MPEG-4 AVC standard were surveyed. In January 2005, at the next meeting of VCEG, VCEG began designating certain topics as "Ke/ (T)

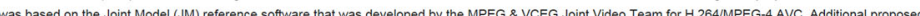
technologies were integrated into the KTA soffware and tested in experiment evaluations over the next four years. ${ }^{[6]}$ Two approaches for standardizing enhanced compression technology were considered: etther creating a new standard or creating extensions of H.264/MPEG-4 AVC. ${ }^{[7]}$ The project had tentative names H.265 and H.NGVC (Next-generation Video Coding), and was a major part of the work of VCEG until its evolution into the HEVC joint project with MPEG in 2010. [IIIII9!)

The preliminary requirements for NGVC was the capability to have a bit rate reduction of $50 \%$ at the same subjective image quality compared to the H.264/MPEC 4 AVC High profile and computational complexity ranging from $1 / 2$ to 3 times that of the High profilie. NGVC would be able to provide $25 \%$ bit rate reduction along with $50 \%$ reduction in complexity at the same perceived video quality as the High profile, or to provide greater bit rate reduction with somewhat higher complexity

Fig. 10 An example image output with normal split mode (video + text)

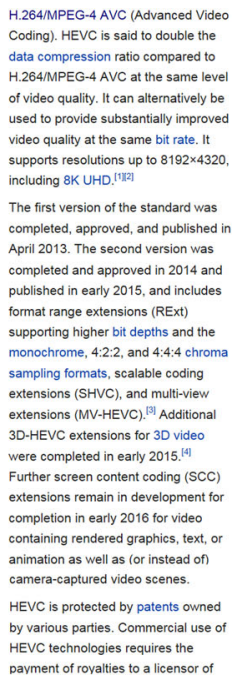

Coding) HEVC is Sadvanced Video data compression ratio compared to of video quality. It can alternatively be used to provide substantially improved supports resolutions up to $8192 \times 4320$,

The first version of the standard was April 2013. The second and published in completed and approved in 2014 and published in early 2015, and includes supporting higher bit depths and the monochrome, 4:2:2, and 4:4:4 chroma extensions (SHVC), and muttiviev' extensions (MV-HEVC). Additiona 3D-HEVC extensions for $3 \mathrm{D}$ vid Further screen content coding ( $\mathrm{SCC}$ ) completetion in early 2016 for video containing rendered graphics, text, or animation as well as (or instead of payment of rovalties to a licensor o 
Table 2 Adjustment cases of the QLD process for 1L/2L BRC algorithm

\begin{tabular}{|c|c|c|c|c|c|}
\hline Number & State & Each mode & LR results & QL1 & QL2 \\
\hline 1 & Stable state $(3.5<\mathrm{LR}<4.5)$ & $1 \mathrm{~L}$ mode & $\mathrm{LR} 1<\mathrm{TCR}$ & +1 & +2 \\
\hline 2 & & & $L R 1>T C R$ & -1 & 0 \\
\hline 3 & & $2 \mathrm{~L}$ mode & $\mathrm{LR} 2<\mathrm{TCR}$ & +2 & +1 \\
\hline 4 & & & $L R 2>T C R$ & 0 & -1 \\
\hline 5 & Scene change state & $1 \mathrm{~L}$ mode & & BRC table1 new QL1 & $\mathrm{QL} 2 \leq \mathrm{QL} 1$ \\
\hline 6 & & $2 \mathrm{~L}$ mode & & $\mathrm{QL} 1 \leq \mathrm{QL} 2$ & BRC table2 new QL2 \\
\hline
\end{tabular}

$L R$ line compression ratio, $T C R$ target compression ratio, $Q L$ quantization level

lines should be increased rapidly. In the remaining line process (RLP) stage of the proposed $1 \mathrm{~L} / 2 \mathrm{~L}$ BRC, if the CR is less than TCR after $70 \%$ lines are processed, the $\mathrm{QL}$ value for the current compression mode is increased by 3 while the QL value for the other compression mode is increased by 4 . Table 3 shows how the adjustment works.

\subsubsection{Split mode processing based on average H1 band energy}

The proposed BRC method can improve the consistency of image quality over the whole frame based on temporal similarity even if the frame is composed of several different images in split mode. The proposed method uses average energy of $\mathrm{H} 1$ band to find image characteristics for low-complexity prediction in time domain, so that it does not require frame memory or motion estimation techniques. The $\mathrm{H} 1$ band contains the highest frequency characteristics among sub-bands, and the higher the average energy of the $\mathrm{H} 1$ band, the lower the compression ratio. Therefore, the energy of $\mathrm{H} 1$ band can be used for image classification for BRC control. For example, a complex texture image such as text lines has higher average energy in the $\mathrm{H} 1$ band than a low texture image such as simple figure does.

In the proposed BRC, a frame is only divided into two parts for simplicity if the frame is in split mode. If the border line between these two parts is located at the top of the frame, there is plenty of room to adjust the compression ratio. On the other hand, if the border line is located at the bottom of the frame, there is a slight chance to enhance the image quality. Therefore, the proposed BRC checks the split mode only in the middle half of the frame. Figure 11 depicts the operation concept of the TCR re-adjustment method in split mode. BRC does not check split mode in the first or the last quarter of the frame. If a large difference of $\mathrm{H} 1$ band's average

Table 3 Adjustment in RLP process for 1L/2L BRC algorithm

\begin{tabular}{llllll}
\hline Number & Q-type & Each mode & CR & QL1 & QL2 \\
\hline 1 & Normal & 1L mode & CR $<$ TCR & +3 & +4 \\
2 & & 2L mode & & +4 & +3 \\
\hline
\end{tabular}

$C R$ cumulative compression ratio, $T C R$ target compression ratio energy is detected within the region of interest (ROI) (second and third quarters), the frame in split mode, location of the border, energy difference, TCR and CR of the upper region (TCR1 and CR1), and TCR and CR of the lower region (TCR2 and CR2) are recorded.

For the next frame, recorded TCR1 and TCR2 are respectively used for the upper and lower regions because of temporal similarity of consecutive frames. If similar energy difference to the previous frame is detected around the location of the border line of the previous frame, it is regarded that the same split mode continues.

The location of the border line can move up or down due to scrolling. Thus, the lower region of the next frame starts as soon as the border line of the next frame is detected. Equation (1) is used to determine TCR2, where HEIGHT is the frame height and $i$ is the length from the first line of the frame to the border line.

$$
\mathrm{TCR} 2=\frac{\text { HEIGHT }-i-1}{\left(\frac{\mathrm{HEIGHT}}{4.05}\right)-\left(\frac{i-1}{\mathrm{CR} 1}\right)}
$$

\subsubsection{Frame unit screen transition process based on $\mathrm{H} 1$ band energy difference values (between frames)}

The proposed BRC method can handle split mode in a single frame. It can also handle scene change between consecutive frames. The scene change is detected if the average energy of $\mathrm{H} 1$ band is changed more than a threshold value $(\mathrm{TH})$ before the last quarter of the frame. If frame unit scene change occurs, compression for remaining regions is performed using default TCR value (4.00). This method can also prevent unnecessary image quality degradation due to scene changes between frames with split mode.

\section{Results and discussion}

To compare energy consumption of multimedia IoT devices with different compression algorithms, energy complexity method [33, 34] based on the number of operations in the microprocessor and the number of 


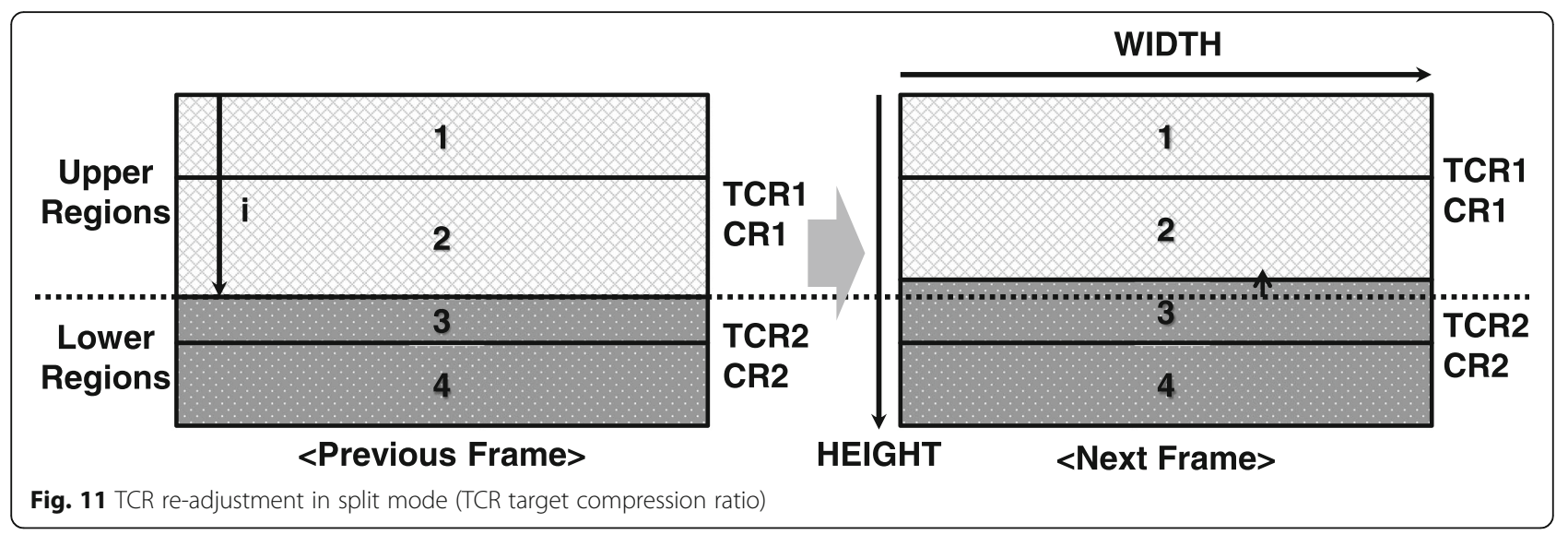

memory accesses can be used. This method can estimate the power consumption of an algorithm operating on a target embedded system while avoiding significant estimation deviation due to many complicated interactions among the embedded program implementation techniques, the optimization level, and the operating system (OS) programs [35]. The energy complexity method can estimate the trend of the energy cost change for multiple algorithms with high accuracy at the theoretical algorithm proposal stage.

To assess power reduction performance of existing IoT systems, we assumed that the evaluation system had specifications similar to those of Intel Galileo gen 2 development board [28] frequently used for commercial purposes. To estimate energy saving effects of the proposed method and existing compression methods, transmission power reduced by compression and processing power for compression based on power consumption characteristics of the evaluation system were considered simultaneously.

First, numbers of computational operations for each compression method and memory access counts were evaluated to predict the power consumption required for compression in terms of each compression algorithm. To predict the amount of transmission power saved due to compression, average compression ratio at visually lossless condition of at least $40 \mathrm{~dB}$ for each compression method was assessed. Computational power consumptions of all algorithms were simulated on a desktop computer using $\mathrm{C}$ programming language, and then, transmission power savings were calculated. Frame compression ratio (FCR), the compression ratio of the whole single frame, was used for compression performance evaluation for each algorithm as shown in Eq. (2).
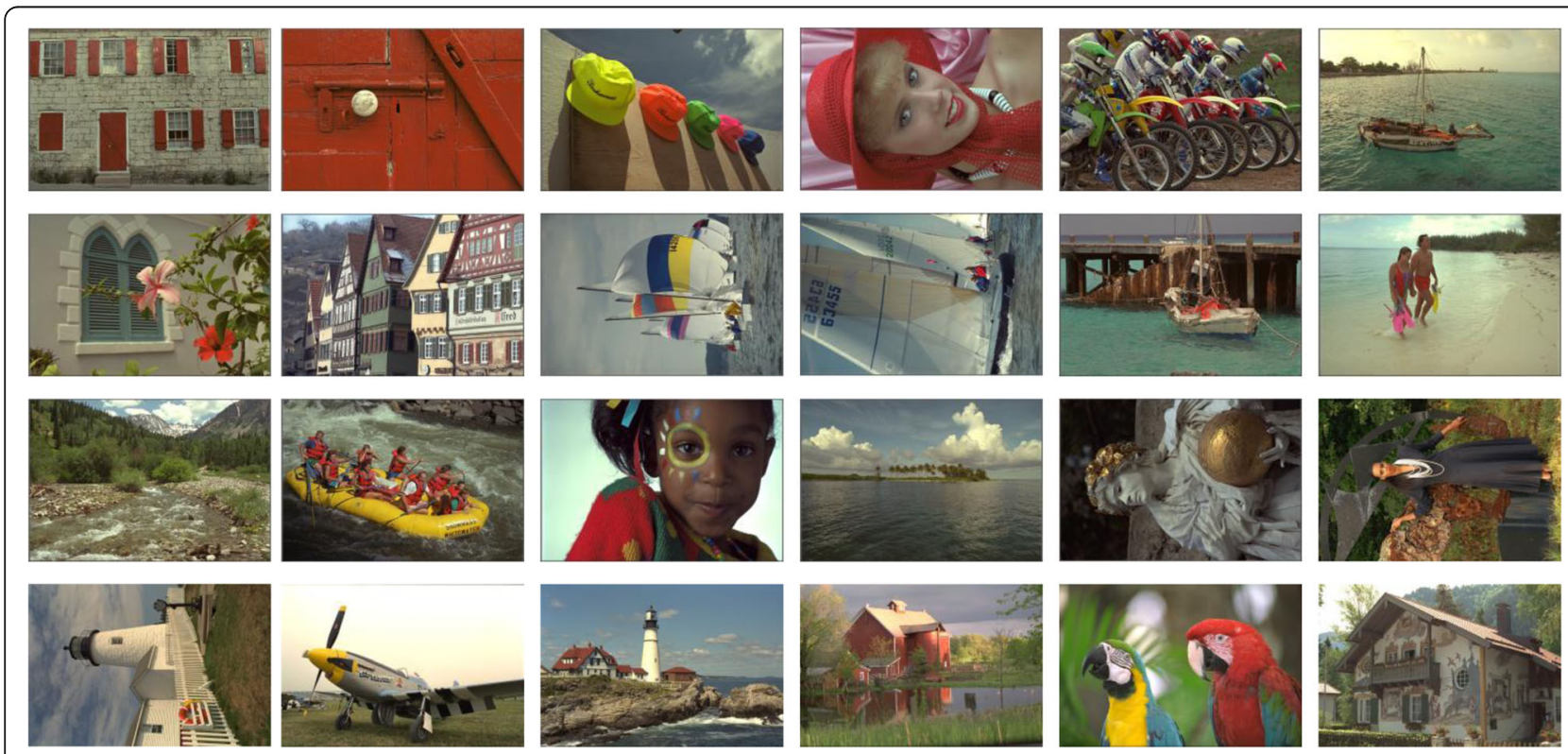

Fig. 12 Twenty-four Kodak still images (image resolution $768 \times 512,512 \times 768$ ) 
Table 4 Energy complexity of each compression algorithm (sum of microprocessor operation and memory accesses)

\begin{tabular}{|c|c|c|c|c|c|c|c|c|c|}
\hline Type & $\begin{array}{l}\text { SPIHT } \\
{[18]}\end{array}$ & $\begin{array}{l}\text { L-BCWT } \\
\text { [23] }\end{array}$ & $\begin{array}{l}\text { ZM-SPECK } \\
{[25]}\end{array}$ & $\begin{array}{l}\text { VLC_NUQ } \\
{[26]}\end{array}$ & $\begin{array}{l}\text { FALC } \\
{[27]}\end{array}$ & $\begin{array}{l}\text { Proposed } \\
\text { method }\end{array}$ & $\begin{array}{l}\text { 8L JPEG } \\
{[40]}\end{array}$ & $\begin{array}{l}\text { 4L H.264 } \\
{[13]}\end{array}$ & $\begin{array}{l}\text { 4L HEVC } \\
{[15]}\end{array}$ \\
\hline $\begin{array}{l}\text { \#CPU operations } \\
\text { (energy cost/frame) }\end{array}$ & $\begin{array}{l}25 \times 3 \mathrm{MN} \\
(14.7)\end{array}$ & $\begin{array}{l}15 \times 3 \mathrm{MN} \\
(8.8)\end{array}$ & $\begin{array}{l}13.9 \times 3 \mathrm{MN} \\
(8.1)\end{array}$ & $\begin{array}{l}38 \times 3 M N \\
(22.4)\end{array}$ & $\begin{array}{l}16 \times 3 M N \\
(9.4)\end{array}$ & $\begin{array}{l}58 \times 3 \mathrm{MN} \\
(34.2)\end{array}$ & $\begin{array}{l}277.6 \times 3 M N \\
(163.7)\end{array}$ & $\begin{array}{l}548 \times 3 M N \\
(323)\end{array}$ & $\begin{array}{l}653 \times 3 \mathrm{MN} \\
(385)\end{array}$ \\
\hline $\begin{array}{l}\text { \#Memory accesses } \\
\text { (energy cost/frame) }\end{array}$ & $\begin{array}{l}15.5 \times 3 M N \\
(10.2)\end{array}$ & $\begin{array}{l}9.5 \times 3 \mathrm{MN} \\
(6.2)\end{array}$ & $\begin{array}{l}12 \times 3 \mathrm{MN} \\
(7.9)\end{array}$ & $\begin{array}{l}9 \times 3 \mathrm{MN} \\
(5.9)\end{array}$ & $\begin{array}{l}6.5 \times 3 \mathrm{MN} \\
(4.2)\end{array}$ & $\begin{array}{l}43.6 \times 3 M N \\
(28.8)\end{array}$ & $\begin{array}{l}37.4 \times 3 M N \\
(24.7)\end{array}$ & $\begin{array}{l}194 \times 3 M N \\
(128)\end{array}$ & $\begin{array}{l}334 \times 3 M N \\
(221)\end{array}$ \\
\hline $\begin{array}{l}\text { Average FCR } \\
\text { (RF energy cost/frame) }\end{array}$ & 2.04:1 (469) & $\begin{array}{l}2.04: 1 \\
(469)\end{array}$ & 2.37:1 (404) & $3.25: 1(295)$ & $3.37: 1(284)$ & 4.9:1 (195) & 11.47:1 (83.4) & 10.49:1 (91) & $12.5: 1(76.5)$ \\
\hline Transmission duration (s) & 0.401 & 0.401 & 0.345 & 0.252 & 0.243 & 0.167 & 0.071 & 0.078 & 0.065 \\
\hline $\begin{array}{l}\text { Total energy cost } \\
(\mathrm{mJ} / \text { frame })\end{array}$ & 494.2 & 484.4 & 420 & 322.9 & 297.8 & 258.4 & 271.9 & 542.6 & 682.4 \\
\hline
\end{tabular}

$M$ image width size, $N$ image height size. CPU operation energy cost, $62.5 \mathrm{pJ} / \mathrm{bit}$; memory access energy cost, $70 \mathrm{pJ} / \mathrm{bit}$; average RF power consumption, $1170 \mathrm{~mW}$; and RF bandwidth, 11 Mbps

For test image, 24 Kodak still images [36] frequently used for image compression evaluations were employed (see Fig. 12).

$$
\text { FCR }=\frac{\text { Number of bits for original image }}{\text { Number of bits for compressed image }}
$$

\subsection{Comparison of power consumption changes}

The power consumption cost of the whole IoT platform [28] was estimated in terms of Intel quark SoC X1000 processor and mobile DRAM memory usage based on energy per bit information [37, 38]. Additionally, radio frequency (RF) power consumption cost for data transmission was estimated with Broadcom BCM43340 [39] known to consume approximately $1170 \mathrm{~mW}$ of average transmission power for $11 \mathrm{Mbps}$ wireless transmission. Since the data size for three-color channels of a single uncompressed image is $9,437,184$ bits $(3 \times 768 \times 512 \times 8)$, the total transmission energy for a single uncompressed image is $957 \mathrm{~mJ}$. Table 4 depicts power consumption required for compression processing for each algorithm and average compression ratio at visually lossless condition. Figure 13 depicts frame compression ratio (FCR) for each compression method. The 1D SPIHT method [18] had the lowest compression ratio of 2.04:1, indicating the lowest reduction in transmission time. It consumed $469 \mathrm{~mJ}$ for RF transmission. It also showed high-power consumption cost due to iterative memory accesses. The L-BCWT method [23] reduced SPIHT's memory access cost. However, its compression ratio was the same as that of $1 \mathrm{D}$ SPIHT. Its RF transmission energy was still $469 \mathrm{~mJ}$. The ZM-SPECK showed higher FCR of 2.37:1 than L-BCWT. However, the achieved compression ratio was not high enough to reduce RF power consumption. Its RF transmission energy was $404 \mathrm{~mJ}$. The FALC method [27] further enhanced compression ratio to $3.37: 1$, with RF transmission energy of $284 \mathrm{~mJ}$. The $8 \mathrm{~L}$ JPEG method is the same as the existing JPEG standard [40] except that it only uses eight lines at a time. The 8L JPEG method had less memory access cost of $37.4 \mathrm{~mJ}$ with higher FCR of 11.47:1 comparing to the proposed method. However, to meet high computational accuracy required for visually lossless image quality, the $8 \mathrm{~L}$ JPEG consumed $163.7 \mathrm{~mJ}$ for floating-point operations [41], which was $13.5 \mathrm{~mJ}$ higher in total energy consumption compared to the proposed method. Although the 4L HEVC method [15] had the highest compression ratio of $12.5: 1$, it required extremely high-power consumption due to heavy memory accesses and computational processing. The 4L H.264 method [13] required lower power consumption than the 4L HEVC method. However, it needed higher power consumption than SPIHT-based methods.

Although the proposed compression method used only one additional line memory and slightly higher operation counts than the FALC method, it achieved better

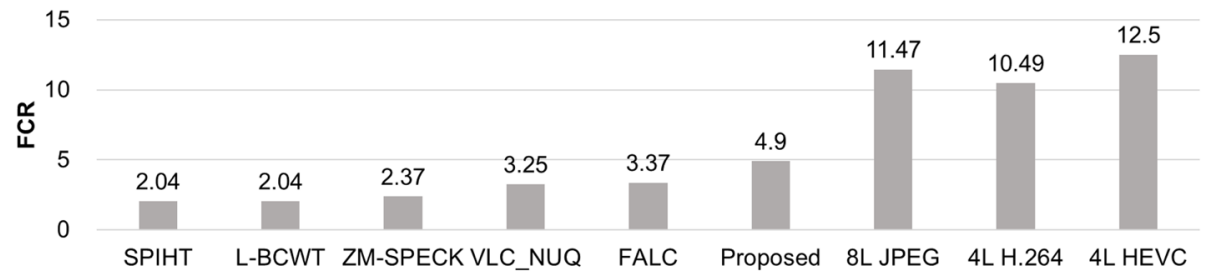

Fig. 13 Average compression ratio results for each compression method (CR compression ratio) 
Table 5 Frame compression ratio and PSNR of bit rate control techniques for Kodak still images [36] (TCR: 4.0)

\begin{tabular}{|c|c|c|c|c|c|c|}
\hline \multirow[t]{2}{*}{ Algorithm } & \multicolumn{2}{|l|}{ Best } & \multicolumn{2}{|l|}{ Worst } & \multicolumn{2}{|c|}{ Average } \\
\hline & PSNR & FCR & PSNR & FCR & PSNR & FCR \\
\hline Proposed & 46.71 & 4.00 & 33.93 & 4.02 & 41.39 & 4.02 \\
\hline Edirisinghe's [29] & 46.54 & 4.01 & 29.11 & 4.06 & 39.77 & 4.03 \\
\hline Jiang's [30] & 45.83 & 4.43 & 33.91 & 4.05 & 40.36 & 4.37 \\
\hline
\end{tabular}

FCR frame compression ratio

compression ratio of 4.9:1 than the VLC_NUQ [26] or the FALC method. The RF transmission energy of the proposed method was reduced to $195 \mathrm{~mJ}$. Furthermore, the proposed method consumed less energy for processing units because of its simple implementation. Based on these comparison results, the proposed method showed the best performance among these methods tested in terms of energy efficiency.

\subsection{BRC tests for split mode in a still image and scene changes in an image sequence}

The proposed bit rate control method and existing techniques were compared in terms of image quality while maintaining target compression ratio at 4.0 sharp. For a fair comparison, existing bit rate control techniques were implemented on top of the proposed compression method. Still images and image sequences were fed to algorithms under test to evaluate the consistency in image quality. Twenty-four Kodak still test images [36] were selected for BRC experiments (see Fig. 12). Experimental image sequences were composed of several merged images of different characteristics to evaluate image quality consistency in split mode with scene changes. These images were obtained from HEVC common test sequences [42] and overlaid on the text image.

Table 5 shows frame compression ratio (FCR) and PSNRs of the bit rate control technique for Kodak still images [36]. The average PSNR of Edirisinghe's BRC [29] was $39.77 \mathrm{~dB}$ with frame compression ratio of 4.03 . The average PSNR of Jiang's BRC [30] was $40.36 \mathrm{~dB}$ with an average FCR of 4.37 which was too high because the target compression ratio was 4.0. Such high compression ratio of Jiang's BRC method [30] caused severe imbalance of image quality in one frame. The frame compression ratio of the proposed BRC was 4.02 which was the closest to TCR of 4.0. The average PSNR of the proposed $\mathrm{BRC}$ was $41.39 \mathrm{~dB}$ which was higher than that of Edirisinghe's BRC [29] and Jiang's BRC [30] by $1.62 \mathrm{~dB}$ and $1.03 \mathrm{~dB}$, respectively.

Figure 14 depicts image sequences for testing image quality consistency. The image sequence was composed of four consecutive images and a scene change in the middle. The t0 t3 indicate sequence numbers of image sequences while R0 R3 denote four equally divided regions of an image from the top to the bottom. Edrisinghe's BRC [29] failed to achieve target compression ratio in $\mathrm{t} 0$, $\mathrm{t} 2$, or $\mathrm{t} 3$. In the event of scene change in t2, PSNRs in regions R2 and R3 were very low, especially in the text region (Fig. 14).

BRC results for test image sequences are shown in Table 6. Jiang's BRC [30] excessively compressed test images due to failures in compression ratio prediction. Consequently, image qualities in $\mathrm{t} 2$ and $\mathrm{t} 3$ were severely
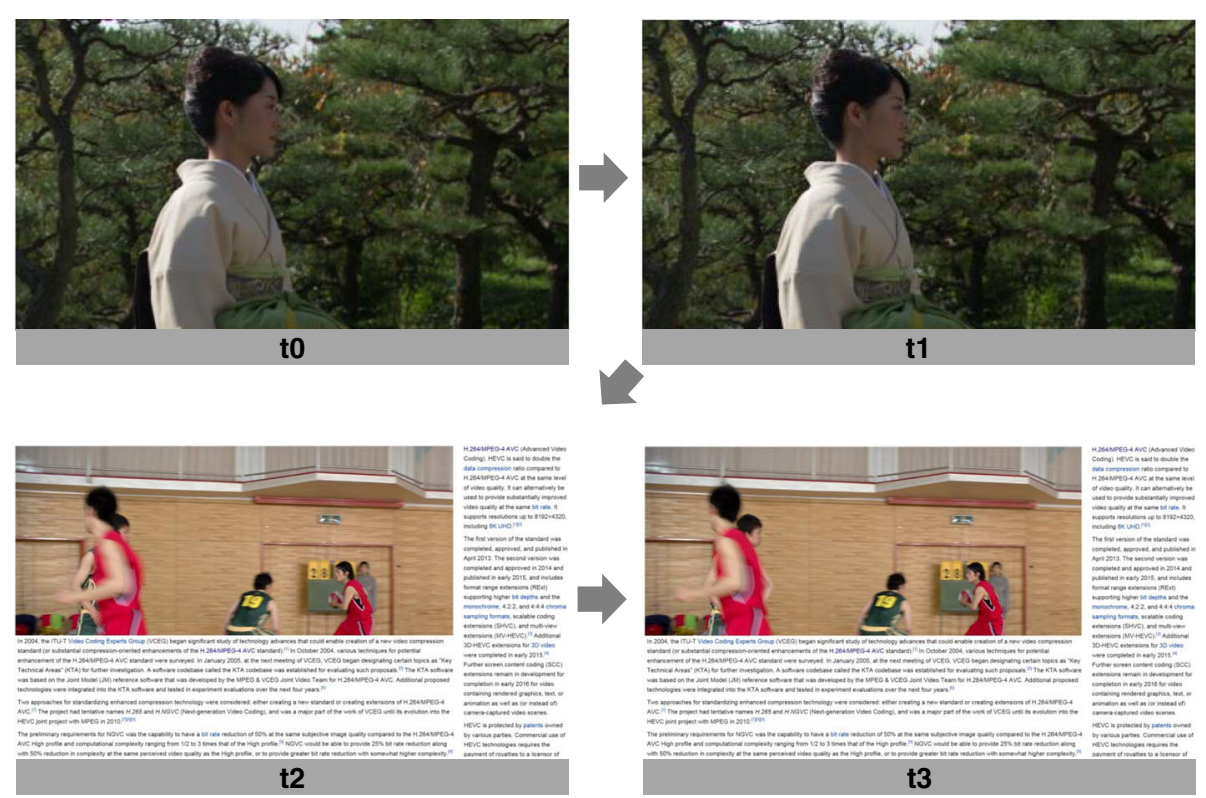

Fig. 14 Image sequences to test image quality consistency (Kimono $1920 \times 1080$, BasketballDrive + Text $1920 \times 1080$ ). t2 shown in Fig. 9 
Table 6 BRC results for testing image sequences

\begin{tabular}{llllllllll}
\hline BRCs & Seq. & TCR1 & TCR2 & R0 & R1 & R2 & R3 & Total & FCR \\
\hline Proposed & t0 & 4.00 & & 43.85 & 44.24 & 44.40 & 43.33 & 43.93 & 4.00 \\
& t1 & 4.05 & & 43.95 & 44.23 & 44.42 & 42.97 & 43.85 & 4.00 \\
& t2 & 4.05 & 4.08 & 42.35 & 42.56 & 38.38 & 36.21 & 39.04 & 4.08 \\
& t3 & 4.55 & 4.00 & 41.26 & 41.36 & 37.84 & 41.06 & 40.10 & 4.03 \\
Edirisinghe's [29] & t0 & 4.00 & & 43.15 & 43.83 & 43.95 & 44.80 & 43.89 & 3.99 \\
& t1 & 4.00 & & 43.35 & 43.30 & 44.59 & 44.28 & 43.84 & 4.01 \\
& t2 & 4.00 & & 42.43 & 42.20 & 20.54 & 22.49 & 24.37 & 3.96 \\
Jiang's [30] & t3 & 4.00 & & 42.58 & 42.30 & 25.24 & 36.94 & 30.82 & 3.95 \\
& t0 & 4.00 & & 41.84 & 40.47 & 41.82 & 43.62 & 41.79 & 5.16 \\
& t1 & 4.00 & & 42.27 & 40.87 & 42.17 & 43.64 & 42.13 & 4.99 \\
& t2 & 4.00 & & 41.80 & 37.64 & 35.82 & 31.87 & 35.41 & 5.24 \\
& t3 & 4.00 & & 40.81 & 38.01 & 34.96 & 31.88 & 35.19 & 5.41 \\
\hline
\end{tabular}

damaged and their PSNRs were decreased to approximately $30 \mathrm{~dB}$.

On the other hand, the proposed BRC maintained a PSNR of $\mathrm{t} 2$ even under scene change condition. PSNRs of the proposed BRC for regions R2 and R3 in image t2 were $38.38 \mathrm{~dB}$ and $36.21 \mathrm{~dB}$, respectively. PSNRs for regions $\mathrm{R} 2$ and $\mathrm{R} 3$ in t3 image sequence were $37.84 \mathrm{~dB}$ and $41.06 \mathrm{~dB}$, respectively. These results showed that the proposed method outperformed existing techniques in terms of PSNR. Moreover, legibility of the text region of the proposed BRC was superior to that of existing BRC techniques as shown in Fig. 15.

\section{Conclusions}

In this paper, a compression method for power reduction in multimedia IoT environments with bit rate control was proposed. The proposed method uses a low-complexity algorithm with the least possible amount of processes and memory access in consideration of IoT environment's limited power supply.

Based on our test results, the proposed method achieved higher compression ratio than existing methods while maintaining lower complexity. It demonstrated superior performance compared to existing SPIHT or $4 \mathrm{~L}$ HEVC method in terms of power reduction effect at system level. It can maintain better restorative and consistent image quality performance in situations with high/low quality imbalance, image split mode, and scene changes that often occur in existing line compression methods.

Future studies include optimization for the system implementation and transmission error recovery techniques to extend the proposed technique to various IoT applications.

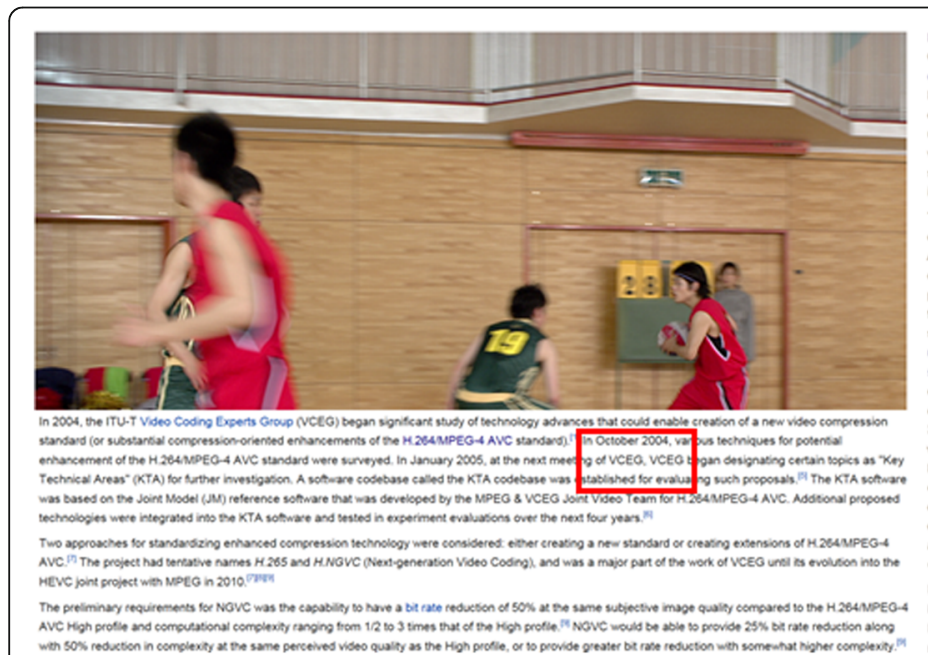

(a)

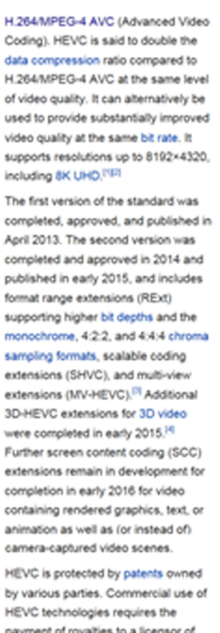

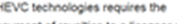

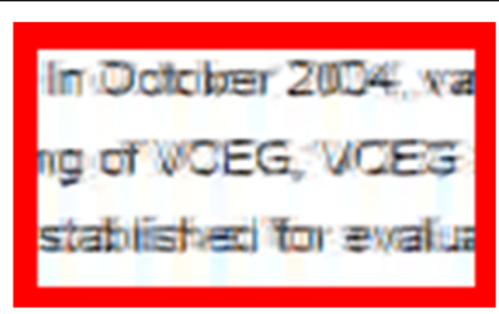

(b)

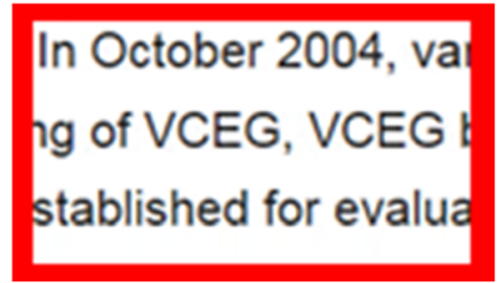

(c)

Fig. 15 Results of text line images in split mode. a Input image. b Edrisinghe's [29] result. c Proposed BRC's result 


\section{Abbreviations}

$1 \mathrm{~L}$ mode: One-line compression mode; 1D: One-dimensional; $2 \mathrm{~L}$ mode: Twoline compression mode; 2D: Two-dimensional; BRC: Bit rate control; CABAC: Context-adaptive arithmetic coding; CR: Compression ratio; DPCM: Differential pulse code modulation; DWT: Discrete wavelet transform; ECG: Electrocardiogram; FALC: Frequency adaptive line compression; FCEE: Frequency component entropy encoding; FCR: Frame compression ratio; HM: HEVC test model; HVS: Human visual system; loT: Internet of things; JM: Joint model; L-BCWT: Line-based backward coding of wavelet trees; LR: Line compression ratio; MED: Median edge detection; OS: Operating system; PSNR: Peak signal-to-noise ratio; QL: Quantization level; QLD: Quantization level determination; RF: Radio frequency; RLP: Remaining line process; ROI: Region of interest; SAD: Sum of absolute difference; SPIHT: Set partitioning in hierarchical trees; TCRs: Target compression ratios; TH: Threshold value; uProc: Microprocessor; VLC: Variable length coding; VLC_NUQ: Visually lossless compression non-uniform quantization; VLSI: Very large-scale integration; ZM-SPECK: Zero memory set partitioned embedded block; zRLE: Zero run-length encoding

\section{Acknowledgements}

SW Lee and HY Kim are co-first authors. They contributed equally to this study. The work reported in this paper was conducted during the sabbatical year of Kwangwoon University in 2018.

\section{Funding}

This work was partly supported by a grant (2016-0-00421) of Institute for Information \& Communications Technology Promotion (IITP) funded by the Korea government (MSIP). It was also supported by a grant (2017R1D1A1B03036361) of the Basic Science Research Program through the NRF (National Research Foundation) of Korea funded by the Ministry of Education, a grant (10080649) funded by the MOTIE (Ministry of Trade, Industry \& Energy), and KSRC (Korea Semiconductor Research Consortium) support program for the development of future semiconductor device.

\section{Availability of data and materials}

The conclusion and comparison data of this article are included within the article.

\section{Authors' contributions}

HY implemented the proposed algorithm, carried out all experiments, and drafted the manuscript. SL conceived the study, designed the proposed algorithm and experiments, and helped draft the manuscript. All authors read and approved the final manuscript.

\section{Authors' information}

Seong-Won Lee: He received B.S. and M.S. degrees in Control and Instrumentation Engineering from Seoul National University, Korea, in 1988 and 1990, respectively. He obtained Ph.D. degree in Electrical Engineering from University of Southern California, Los Angeles, CA, in 2003. From 1990 to 2004, he worked on VLSI/ System-on-Chip (SoC) design at Samsung Electronics Co., Ltd., Korea. Since 2005, he has been a Professor in Computer Engineering Department, Kwangwoon University, Seoul, Korea. His research interests include image signal processing, signal processing SoC, and computer architecture.

Ho-Young Kim: He received B.S. and M.S. degrees in Computer Engineering from Kwangwoon University, Seoul, Korea, in 2011 and 2013, respectively. He is currently pursuing a Ph.D. degree in Computer Engineering at Kwangwoon University, Seoul, Korea. His research interests include image signal processing, signal processing SoC, and energy management system.

\section{Competing interests}

The authors declare that they have no competing interests.

\section{Publisher's Note}

Springer Nature remains neutral with regard to jurisdictional claims in published maps and institutional affiliations.
Received: 29 December 2017 Accepted: 7 September 2018 Published online: 17 September 2018

\section{References}

1. M.C. Kang, S.H. Chae, J.Y. Sun, S.H. Lee, S.J. Ko, An enhanced obstacle avoidance method for the visually impaired using deformable grid. IEEE Trans. Consum. Electron. 63(2), 169-177 (2017)

2. J. Ruminski, A. Bujnowski, T. Kocejko, J. Wtorek, A. Andrushevich, M. Biallas, R. Kistler, Performance analysis of interaction between smart glasses and smart objects using image-based object identification. Int. J. Distributed Sensor Networks 12(3), 1-14 (2016)

3. H. Qian, J. Huang, L. Ma, The design challenges for unmanned vehicular video streaming. in Proc. Int. Conf. Vehicular Electronics and Safety (ICVES), 19-24 (2011)

4. A. Tikanmaki, T. Bedrnik, R. Raveendran, J. Roning, The remote operation and environment reconstruction of outdoor mobile robots using virtual reality. in Proc. Int. Conf. Mechatronics and Automation (ICMA), 1526-1531 (2017)

5. S.L. Chen, T.Y. Liu, C.W. Shen, M.C. Tuan, VLSI implementation of a costefficient near-lossless CFA image compressor for wireless capsule endoscopy. IEEE Access 4, 10235-10245 (2016)

6. A. Mammeri, B. Hadjou, A. Khoumsi, A survey of image compression algorithms for visual sensor networks. J. ISRN Sensor Networks 2012(760320), 1-19 (2012)

7. T. Ma, M. Hempel, D. Peng, H. Sharif, A survey of energy-efficient compression and communication techniques for multimedia in resource constrained systems. IEEE Commun. Surveys and Tutorials 15(3), 963$972(2013)$

8. H. ZainEldin, M. Elhosseini, H. Ali, Image compression algorithms in wireless multimedia sensor networks: a survey. Int. J. Ain Shams Engineering 6(2), 481-490 (2015)

9. F. Walls, A. MacInnis, VESA display stream compression for television and cinema applications. IEEE J. Emerging and Selected Topics in Circuits and Systems 6(4), 460-470 (2016)

10. J.I. Odagiri, Y. Nakano, S. Yoshida, Video compression technology for invehicle image transmission: SmartCODEC. J. FUJITSU Scientific\&Technical 43(4), 469-474 (2007)

11. H.H. Chou, Y.J. Chen, Y.C. Shiau, T.S. Kuo, An effective and efficient compression algorithm for ECG signals with irregular periods. IEEE Trans. Biomedical Engineering 53(6), 1198-1205 (2006)

12. D. Cruz, R. Grosbois, T. Ebrahimi, JPEG 2000 performance evaluation and assessment. Int. J. Signal Process. Image Commun. 17(1), 113-130 (2002)

13. ITU-T and ISO/IEC JTC 1, Advanced video coding for generic audiovisual services. ISO/IEC 14496-10, ITU-T Rec. H.264) Version 1-25 April 2017

14. Joint Video Team (JVT) of ISO/IEC MPEG \& ITU-T VCEG, H.264/AVC reference software JM 19.0, June 2015. http://iphome.hhi.de/suehring/tml. Accessed 22 Dec 2017

15. ITU-T and ISO/IEC, High Efficiency Video Coding, ITU-T Rec. H.265 and ISO/ IEC 23008-2 (HEVC) Version 1-4 Dec. 2016

16. C Rosewarne, B Bross, M Naccari, K Sharman, G Sullivan, High efficiency video coding (HEVC) test model 16 (HM 16) improved encoder description update 2. JCTVC-N15139 Feb 2015

17. L. Lacassagne, D. Etiemble, S.A.O. Kablia, 16-bit floating point instructions for embedded multimedia applications. Paper presented at the 7th International Workshop on Computer Architecture for Machine Perception (CAMP'05), Palermo, Italy, 4-6 July 2005

18. Z. Lu, D. Kim, W. Pearlman, Wavelet compression of ECG signals by the set partitioning in hierarchical trees algorithm. IEEE Trans. Biomed. Eng. 47(7), 849-856 (2000)

19. W. Pearlman, A. Islam, N. Nagaraj, A. Said, Efficient, low-complexity image coding with a set-partitioning embedded block coder. IEEE Trans. Circuits and Systems for Video Technol. 14(11), 1219-1235 (2004)

20. M. Akter, M. Reaz, F. Yasin, F. Choong, A modified set partitioning in hierarchical trees algorithm for real-time image compression. J. Commun. Technol. and Electron. 53(6), 642-650 (2008)

21. R. Senapati, U. Pati, K. Mahapatra, Listless block-tree set partitioning algorithm for very low bit rate embedded image compression. Int. J. Electron. Commun. 66(12), 985-995 (2012)

22. M. Tausif, N. Kidwai, E. Khan, M. Reisslein, FrWF-based LMBTC: memoryefficient image coding for visual sensors. IEEE J. Sensors 15(11), 6218-6228 (2015) 
23. L. Ye, J. Guo, B. Nutter, S. Mitra, Memory-efficient image codec using linebased backward coding of wavelet trees. in Proc. Data Compress. Conf. (DCC), 213-222 (2007)

24. L. Ye, J. Guo, B. Nutter, S. Mitra, Low-memory-usage image coding with linebased wavelet transform. J. Opt. Eng. 50(2), 027005-027001 (2011)

25. N. Kidwai, E. Khan, M. Reisslein, ZM-SPECK: a fast and memoryless image coder for multimedia sensor networks. IEEE J. Sensors 16(8), 2575-2587 (2016)

26. D. Vo, S. Lertrattanapanich, Y.T. Kim, Low line memory visually lossless compression for color images using non-uniform quantizers. IEEE Trans. Consum. Electron. 57(1), 187-195 (2011)

27. H.Y. Kim, J.H. Cho, J. Cho, S.W. Lee, A frequency adaptive line compression system for mobile display devices. J. IEICE Electronics Express 11(19), 20140746 (2014)

28. Datasheet, Intel Galileo Gen 2 Development Board. (Intel Co., 2014), https:// www.rutronik.com/fileadmin/Micropages/Intel/intelgalileogen2prodbrief_ 330736_003.pdf. Accessed 22 Dec 2017

29. E. Edirisinghe, S. Bedi, Variation of JPEG-LS to low cost rate control and its application in region-of-interest based coding. Int. J. Signal Process. Image Commun. 18(5), 357-372 (2003)

30. J. Jiang, A low-cost content-adaptive and rate-controllable near-lossless image codec in DPCM domain. IEEE Trans. Image Processing 9(4), 543-554 (2000)

31. J.S. Ham, H.Y. Kim, S.W. Lee, A consistent quality bit rate control for the linebased compression. IEIE Trans. Smart Processing \& Computing 5(5) (2016)

32. Savithra Eratne, Mahinda Alahakoon, Fast predictive wavelet transform for lossless image compression, in Proc. International Conference on Industrial and Information Systems (ICIIS) (University of Peradeniya, Sri Lanka, 2009) https://ieeexplore.ieee.org/document/5429833/

33. K Zotos, A Litke, A Chatzigeorgiou, S Nikolaidis, G Stephanides, energy complexity of software in embedded systems. in Proc. IASTED Int. Conf. Autom. Control Appl. (ACIT-ACA) (Novosibirsk, 2005)

34. V. Konstantakos, A. Chatzigeorgiou, S. Nikolaidis, T. Laopoulos, Energy consumption estimation in embedded systems. IEEE Trans. Instrum. Meas. 57(4), 797-804 (2008)

35. Tao Li, Lizy Kurian John, Run-time modeling and estimation of operating system power consumption, in Proc. the 2003 ACM SIGMETRICS International Conference on Measurement and Modeling of Computer Systems, (2003), 160-171

36. Kodak Lossless True Color Image Suite. http://rOk.us/graphics/kodak. Accessed 22 Dec 2017

37. Datasheet, Intel Quark SoC X1000 329676-005US (Intel Co., 2015), pp. 69-71, https://www.intel.com/content/dam/www/public/us/en/documents/ datasheets/quark-x1000-datasheet.pdf. Accessed 22 Dec 2017

38. K Malladi, F Nothaft, K Periyathambi, B Lee, C Kozyrakis, M Horowitz, Towards energy-proportional datacenter memory with mobile DRAM. in Proc. IEEE 39th International Symposium on Computer Architecture (ISCA), (2012), pp. 37-48

39. Datasheet, BCM43340. (Broadcom Co., 2015), pp. 135, http://www.mouser. com/ds/2/100/002-14943_0l_V-961661.pdf. Accessed 22 Dec 2017

40. G.K. Wallace, The JPEG still picture compression standard. IEEE Trans. Consum. Electron. 38(1), xviii-xxxiv (1992)

41. Gokul Govindu, Ling Zhuo, Seonil Choi, Padma Gundala, Viktor K Prasanna, Area, and power performance analysis of a floating-point based application on FPGAs, in Proc. 7th Annual Workshop on High Performance Embedded Computing (HPEC 2003) (MIT Lincoln Laboratory, 2003)

42. F Bossen, Common test conditions and software reference configurations. in document JCTVC-L1100 (Geneva, 2013) http://phenix.it-sudparis.eu/jct/ doc_end_user/documents/12_Geneva/wg11/JCTVC-L1100-v1.zip. Accessed 22 Dec 2017

\section{Submit your manuscript to a SpringerOpen ${ }^{\circ}$ journal and benefit from:}

- Convenient online submission

- Rigorous peer review

- Open access: articles freely available online

- High visibility within the field

- Retaining the copyright to your article

Submit your next manuscript at $\boldsymbol{\nabla}$ springeropen.com 